\title{
A LOWER BOUND ON THE NUMBER OF VERTICES OF A GRAPH
}

\author{
V. G. KANE AND S. P. MOHANTY
}

\begin{abstract}
In this note, we derive a lower bound for the number of vertices of a graph in terms of its diameter, $d$, connectivity $k$ and minimum degree $\rho$ which is sharper than that of Watkins [1] by an amount $2(\rho-k)$.
\end{abstract}

Let $G$ be any finite, undirected graph with neither loops nor multiple edges. Let $n, \rho, k$ and $d$ denote the number of vertices, minimum degree, connectivity and diameter of $G$ respectively. Watkins [1] has proved that if $k \geqslant 1$, then $n \geqslant k(d-1)+2$. He has used Menger's theorem to obtain the above result. In this note we prove a theorem from which Watkins' result follows as a corollary. Our proof is simple and elementary. Moreover the lower bound we obtain is sharper than that of Watkins by the amount $2(\rho-k)$.

THEOREM 1. If $k \geqslant 1$, then

$$
n \geqslant\left[\begin{array}{ll}
k(d-3)+2 \rho+2, & \text { if } d \geqslant 3, \\
\rho+2, & \text { if } d=2, \\
2, & \text { if } d=1 .
\end{array}\right.
$$

Proof. Let $a$ and $b$ be two vertices of $G$ at a distance $d$. Let $A_{i}=\{x \in$ $V(G) \mid \delta(a, x)=i\}, i=0,1, \ldots, d$, where $\delta(a, x)$ denotes the length of the shortest path between $a$ and $x$. Clearly, $A_{i} \cap A_{j}=\varnothing$ for $i \neq j, A_{0}=\{a\}$ and $b \in A_{d}$. Let $d \geqslant 3$. If we delete all the vertices in any $A_{i}, 1 \leqslant i \leqslant d-1$ from $G$, then the remaining graph becomes disconnected. Hence $\left|A_{i}\right| \geqslant k$ for $1 \leqslant i \leqslant d-1$. Also $\left|A_{0}\right|+\left|A_{1}\right| \geqslant \rho+1$ and $\left|A_{d-1}\right|+\left|A_{d}\right| \geqslant \rho+1$. Hence $n \geqslant k(d-3)+2 \rho+2$. If $d=2$, then $\left|A_{1}\right| \geqslant \rho$ and so $n \geqslant \rho+2$. If $d=1$, then clearly $n \geqslant 2$. Hence the theorem is proved.

Since $\rho \geqslant k$, we have Watkins' result as a direct corollary to the above theorem.

Now we give below examples of two classes of graphs to show that the bounds in our theorem are 'best possible'.

ExAmple 1 (WATKInS [1]). Let $H_{1}, \ldots, H_{d-1}$ represent disjoint copies of $K_{k}, G$ is formed as follows: Join each vertex of $H_{i}$ to each vertex of $H_{i+1}$ by an edge $(i=1, \ldots, d-2)$; then join a new vertex $u$ to each vertex of $H_{1}$ by an edge and similarly join a vertex $v$ to each vertex of $H_{d-1}$. The resulting

Received by the editors August 1, 1977 and, in revised form, November 23, 1977.

AMS (MOS) subject classifications (1970). Primary $05 C 35$. 
graph clearly satisfies the bounds in the theorem but does not show the sharpness of our bound as we have here $\rho=k$.

ExAmple 2. Let $d$ and $m$ be integers at least 2, and let $G$ be the lexicographic product of the $2 d$-circuit with the complete graph $K_{m}$. Then we have $k=2 m, \rho=3 m-1$ and $n=2 m d$. Now $G$ has diameter $d$ and substitution yields $k(d-3)+2 \rho+2=n$.

In this example we have $\rho>k$ and hence our lower bound is sharper than that of Watkins by an amount $2(\rho-k)$.

ACKNOWLEDGEMENT. We are extremely thankful to the referee for suggesting the class of graphs given in Example 2.

\section{REFERENCES}

1. M. E. Watkins, $A$ lower bound on the number of vertices of a graph, Amer. Math. Monthly 74 (1967), 297.

Electronics Commission, New Delhi, India

Department of Mathematics, Indian Institute of Technology, KanPUR 208016 India 